\title{
In Situ Monitoring of Dispersion Dynamics of Carbon Nanotubes during Sonication Using Electrical Conductivity Measurements
}

\author{
Syed Sadiq Ali, ${ }^{1}$ Mohammed Shahabuddin, ${ }^{2}$ and Mohammad Asif ${ }^{3}$ \\ ${ }^{1}$ Department of Chemical Engineering, King Faisal University, P.O. Box 380, Al-Ahsa 31982, Saudi Arabia \\ ${ }^{2}$ Department of Physics \& Astronomy, King Saud University, P.O. Box 2455, Riyadh 11451, Saudi Arabia \\ ${ }^{3}$ Department of Chemical Engineering, King Saud University, P.O. Box 800, Riyadh 11421, Saudi Arabia \\ Correspondence should be addressed to Mohammad Asif; masif@ksu.edu.sa
}

Received 31 July 2015; Accepted 27 September 2015

Academic Editor: Stefano Bellucci

Copyright ( 2015 Syed Sadiq Ali et al. This is an open access article distributed under the Creative Commons Attribution License, which permits unrestricted use, distribution, and reproduction in any medium, provided the original work is properly cited.

\begin{abstract}
The main challenge in the fabrication of carbon nanotube- (CNT-) based composite materials is the optimization of the sonication time in order to obtain homogenous and uniform dispersion of CNTs. Past studies mostly relied on postprocessing characterization techniques to address this issue. In the present, however, in situ monitoring of dispersion dynamics of CNTs in distilled water is carried out using instantaneous conductivity measurements. Using a computer controlled data acquisition system, the time evolution of the solution conductivity was carefully recorded. The data were then used to evaluate the intensity of turbulent fluctuations, which clearly highlighted the existence of three distinct sonication phases. During the first phase, the conductivity fluctuations initially increased attaining ultimately a maximum, thus indicating the occurrence of large agglomerates of CNTs. During the second phase of sonication, the solution conductivity showed a rather steep increase while fluctuations steadily declined. This phenomenon can be attributed to the breakdown of large CNT agglomerates, resulting in greater dispersion homogeneity of CNTs. During the third phase, after almost $650 \mathrm{~kJ} / \mathrm{L}$ of sonication energy, the conductivity increase was almost negligible. The fluctuation intensity also remained constant during this phase signifying that the further sonication was no longer required.
\end{abstract}

\section{Introduction}

Ever since the discovery of carbon nanotubes (CNTs), their application has opened new avenues of synthesis and development of advanced functional composite materials with their properties carefully engineered to meet specific applications owing to their extremely high strength and unusually high electrical properties and aspect ratio. However, the nanometric dimension and high aspect ratio of CNTs present an important challenge in the fabrication and processing of CNT-based composite materials due to their nonuniform dispersion as a result of agglomeration [1-3]. This phenomenon compromises both the functionality and the reliability of composite material. In fact, a homogeneous and uniform dispersion of CNTs is an important prerequisite for the development of any commercially viable composite material.
The processing methods used for the dispersion of CNTs in a solution or a polymer matrix could either be mechanical [4-6] or chemical. Since chemical methods are based on the chemically induced modification of the CNT surface [79], this could lead to the contamination of the composite material thus prepared. Therefore, mechanical methods, in spite of being highly energy intensive, are generally preferred. Probably, the most important example of mechanical mixing is the ultrasonication process, whereby the electrical energy is converted into mechanical vibrations, thus producing pressure waves. These waves lead to the formation of bubbles, which collapse to create high intensity turbulence thus enhancing the mixing process. Therefore, longer sonication duration usually leads to a corresponding increase in the dispersion and homogeneity of CNTs distribution in the mixture. This, however, consumes higher energy and moreover 
could cause the breakage of high aspect ratio CNTs. While higher energy consumption affects the economic viability of the product, CNT breakage degrades the product's quality. A careful optimization of the sonication duration is therefore necessary for the synthesis and development of high quality and economically viable composite materials.

A number of researchers have addressed the issue of optimum sonication time in the synthesis and development of various polymer-CNT composites. The approach used invariably consists of postprocessing characterization of the synthesized product using different methods such as UVabsorption [10-12], various microscopic imaging methods, for example, SEM, TEM, and AFM [1, 6, 13-16], and the particle size analysis [17]. However sophisticated, these postprocessing methods might not always reflect the true picture of the state of dispersion as CNTs immediately tend to reaggregate as soon as the sonication process is interrupted [18]. Moreover, sample preparation for such characterization studies could also affect the dispersion homogeneity achieved during sonication. Therefore, postprocessing characterization results cannot be reliably used for determining the optimum sonication time. On the other hand, Huang and Terentjev [18] have used rheological characterization in order to determine the extent of dispersion. At regular time intervals during sonication, the sample was taken from the vessel and its viscosity was measured. Though this technique is probably more representative of dispersion homogeneity than postprocessing microscopic measurements mentioned earlier, the extraction of the sample from the sonication vessel and its subsequent rheological characterization, albeit relatively simpler and faster, would always lead to readjustment and reaggregation of CNTs. Moreover, since the sample is extracted from a point in the sonication vessel, the local variation in the CNT agglomeration level in different regions of the vessel could also influence the results especially during the initial stages of sonication. In order to investigate the dispersion behavior of CNTs during sonication in a thermosetting polyester resin, Battisti et al. [19] carried out on-line measurement of electrical resistivity coupled with off-line rheological characterization, which was further supplemented with optical and scanning electron microscopy. The solution was pumped through the resistivity cell and its resistivity was recorded. Resistivity of the solution initially showed a substantial decrease with the progress of sonication. However, energy input beyond $0.5 \mathrm{~kJ} / \mathrm{g}$ contributed little to the resistivity decrease. In order to control temperature in their experiments, the sonicator was switched on and off at regular intervals of time.

In the present work, an in situ monitoring technique is developed in order to accurately characterize the dispersion of CNTs during the process of sonication for determining the optimum sonication time. The proposed experimental strategy consists of monitoring the instantaneous electrical conductivity of a temperature-controlled solution that contains CNTs in distilled water by using conductivity probe connected to a conductivity meter. The analog output of the conductivity meter was recorded by the computer-controlled data acquisition system. The dispersion dynamics of CNTs in the solution will be reflected in the local instantaneous

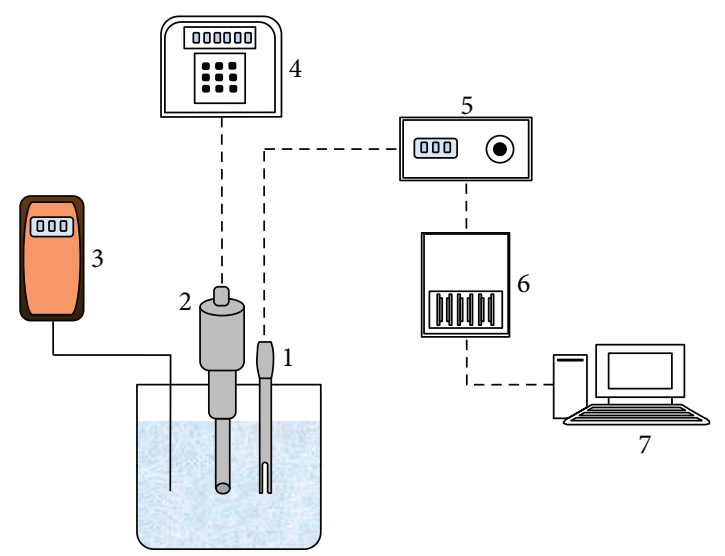

FIGURE 1: Schematic of experimental set-up: (1) conductivity probe; (2) sonicator probe; (3) thermometer; (4) sonicator; (5) conductivity meter; (6) data acquisition system; (7) computer.

electrical conductivity of the solution. A steady value of the conductivity and its fluctuations will indicate the homogeneity of the solution. This means that there is no need to further continue the sonication process. Note that the proposed work is not related to the measurement of thermal conductivity of the nanofluids which appears to be widely discussed and reported in the literature [20].

\section{Experimental}

The present set-up consisted of a $1000 \mathrm{~mL}$ beaker containing the solvent and carbon nanotubes (CNTs). Figure 1 shows the schematic of the experimental set-up. Vibra-Cell VCX 750 (Sonics \& Material, Inc.), ultrasonic processor of $750 \mathrm{~W}$ power and $200 \mathrm{kHz}$ frequency with standard $13 \mathrm{~mm}$ diameter probe, was used for sonication. The solution temperature was carefully monitored in all the experiments. The solvent here was distilled water of conductivity $2 \mu \mathrm{S} / \mathrm{cm}$ at room temperature. The CNT sample used here comprised low aspect ratio short multiwalled carbon nanotubes (s-MWCNT) supplied by Cheap Tubes Inc., USA. Its OD varies from 8 to $15 \mathrm{~nm}$, while the length ranges from 0.5 to $2 \mu \mathrm{m}$. A clear TEM image of the CNT sample can be seen elsewhere [21].

To determine the dispersion dynamics of CNTs in the solvent, in situ measurement of the instantaneous electrical conductivity has been carried out using a conductivity probe connected to a conductivity meter. For accurately monitoring the CNT dispersion dynamics, the analog output voltage signal of the conductivity meter was recorded using a data acquisition system (DAQ) at a sampling frequency of 10 data per second. After carefully examining the design of several conductivity probes, InLab 731 ISM (Mettler Toledo) was chosen here. Its diameter and shaft length were $12 \mathrm{~mm}$ and $120 \mathrm{~mm}$, respectively. The distance between the two electrodes of the conductivity probe was large enough to ensure unhindered flow of solution through the passage.

As a first step in the present experimental strategy, the response of the conductivity measurement system was 


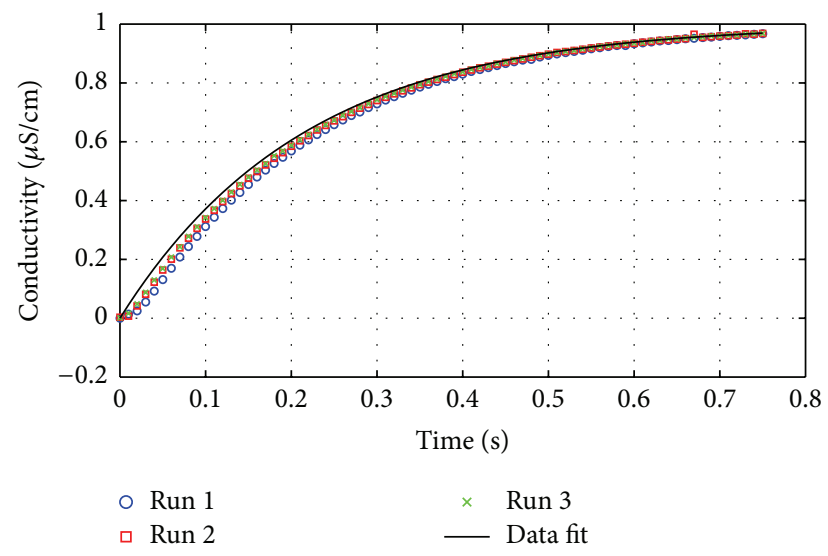

FIGURE 2: Response of the probe for a step change in the electrical conductivity and the data fit using (1).

determined by introducing a step change in the solution conductivity. This is shown in Figure 2. The following equation was used for fitting the experimental data:

$$
C=C_{\infty}\left(1-e^{-t / \tau}\right),
$$

where $C_{\infty}$ is the conductivity of the solution at the steady state. It is clear from the figure that conductivity measurement system exhibits a first-order response with a time constant $(\tau)$ of $0.215 \mathrm{~s}$. The probe calibration was frequently tested using standard conductivity solution during various stages of the present experimental investigation. Additionally, due care was taken to keep electrodes of the probe clean.

Figure 3 shows the location of the conductivity probe and the sonicator inside the sonication vessel. This configuration was kept fixed in all experimental runs. It was found that if the conductivity probe was placed very close to the container wall, the conductivity values did not change with time as shown in Figure 4. This could be attributed to the occurrence of dead zones in the vicinity of the wall. On the other hand, if the conductivity probe is placed near the sonicator, it could damage the probe due to the high cavitation energies.

\section{Methodology}

The CEINT/NIST protocol that deals with the preparation of nanoparticle dispersion from powdered material using ultrasonic disruption was closely followed during experiments [22]. First, $900 \mathrm{~mL}$ of distilled water was taken in the container and a weighted amount of MWCNT was added in it. The sonication vessel was then placed in a temperaturecontrolled water bath. Initially, CNTs were in the form of large flocs, which varied in size ranging from microns to few millimeters. A gentle manual stirring caused it to settle at the bottom of the container owing to its high density. Next, both the conductivity and sonicator probes were dipped in the solution. The conductivity probe was carefully aligned as shown in Figure 3. The conductivity data logging was started before switching on the sonicator. In the present work, the temperature of the solution was maintained at $38 \pm 1^{\circ} \mathrm{C}\left(37^{\circ} \mathrm{C}-\right.$ $39^{\circ} \mathrm{C}$ ) with the help of cooling bath.

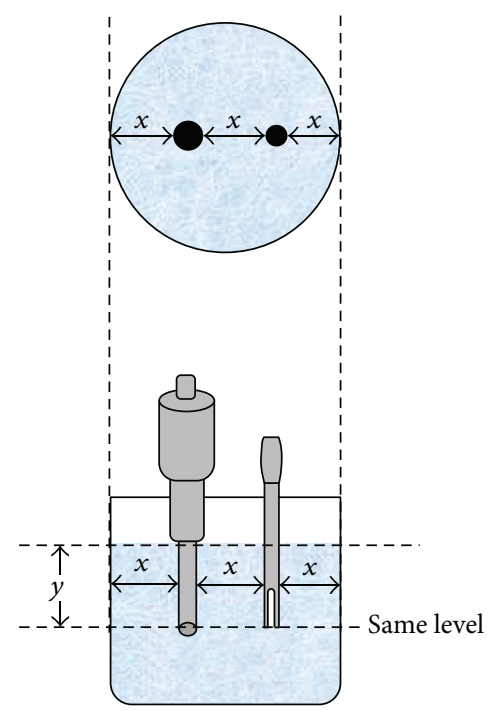

FIGURE 3: Alignment of sonicator probe and conductivity probe in the container.

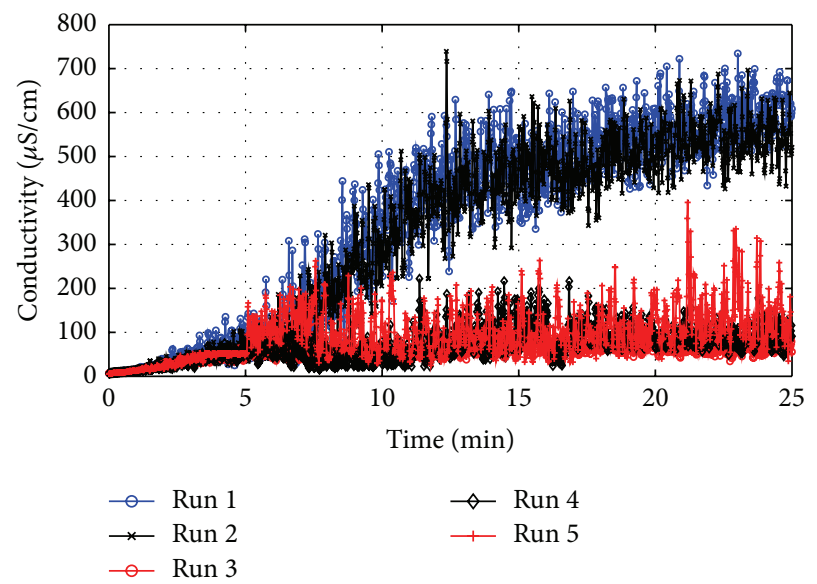

FIGURE 4: Effect of probe alignement on electrical conductivity of $1.11 \mathrm{~g} / \mathrm{L}$ CNT solution. Runs 1-3 are carried out with probe location (as shown in Figure 3), while Runs 4-5 are carried out with conductivity probe located close to the container wall.

Experiments were carried out for three concentrations of CNTs, namely, $1.11 \mathrm{~g} / \mathrm{L}, 0.67 \mathrm{~g} / \mathrm{L}$, and $0.33 \mathrm{~g} / \mathrm{L}$, by keeping the amount of solvent the same at $900 \mathrm{~mL}$ and varying the mass of the CNTs. Several runs were carried out to examine the reproducibility of the data. Fresh s-MWCNT sample was used in each run.

\section{Results and Discussion}

First, the size analysis of as-received CNT sample was carried out using Microtrac particle size analyzer (model S3500). Figure 5 shows the size distribution for three replicate runs of wet analysis of the sample. It is obvious here that CNTs mainly exist as large aggregates with a substantial size distribution. The size of some aggregates in the sample could reach as much as $400 \mu \mathrm{m}$, which is almost 200 times as large as the longest 


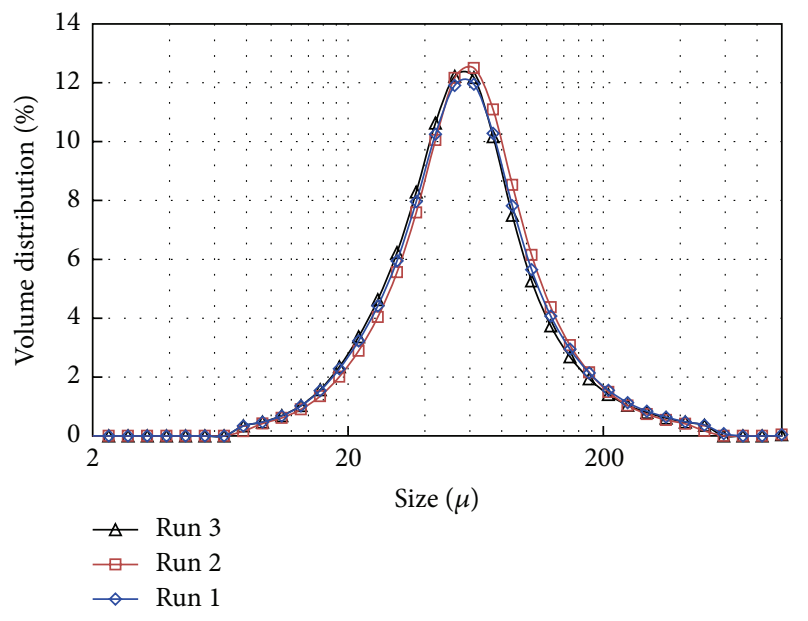

FIGURE 5: Size distribution of CNT aggregates in the as-received sample of short multiwall carbon nanotube (s-MWCNT).

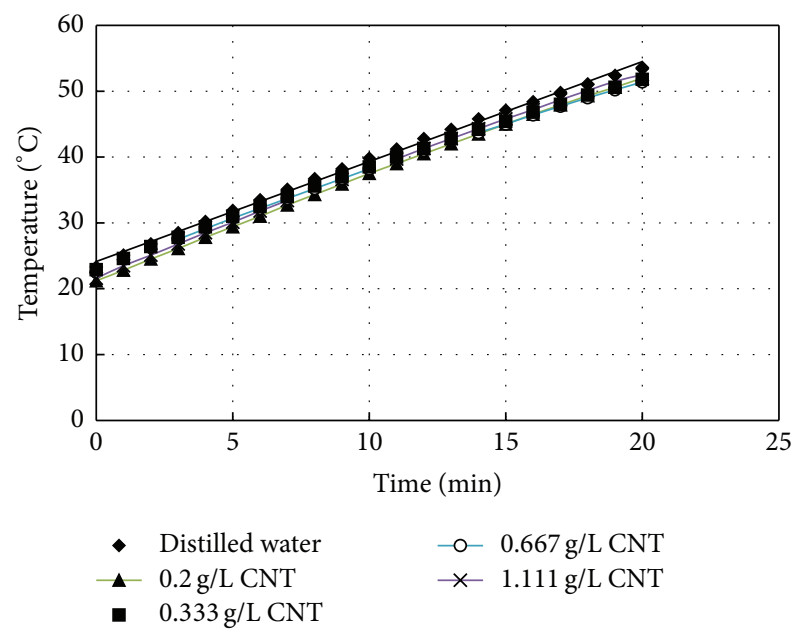

FIGURE 6: Effect of sonication time on temperature of CNT solutions at different concentrations.

CNT present in the sample. For the present sample, volume mean diameters were found to be $68.24,67.69$, and $66.03 \mu \mathrm{m}$, respectively, while standard deviations were $35.35,34.41$, and $33.46 \mu \mathrm{m}$, respectively.

Since optimization of the sonication energy is an important aspect of the present investigation, the part of the sonication energy that is dissipated into heat was also evaluated here. Towards this end, the change in the temperature of the solvent and solutions (solvent + CNTs) for different concentration of CNT with the progress of the sonication process was monitored. This is shown in Figure 6. Although there is a significant rise in the solution temperature with the sonication, the contribution of the CNTs to the heat capacity of the solution nonetheless remains negligible owing to its low concentration. Note that the temperature increase during 5 minutes of sonication is almost $8.6^{\circ} \mathrm{C}$ near ambient conditions when the heat losses to the surrounding could be assumed negligible. Thus, the part of the ultrasonication

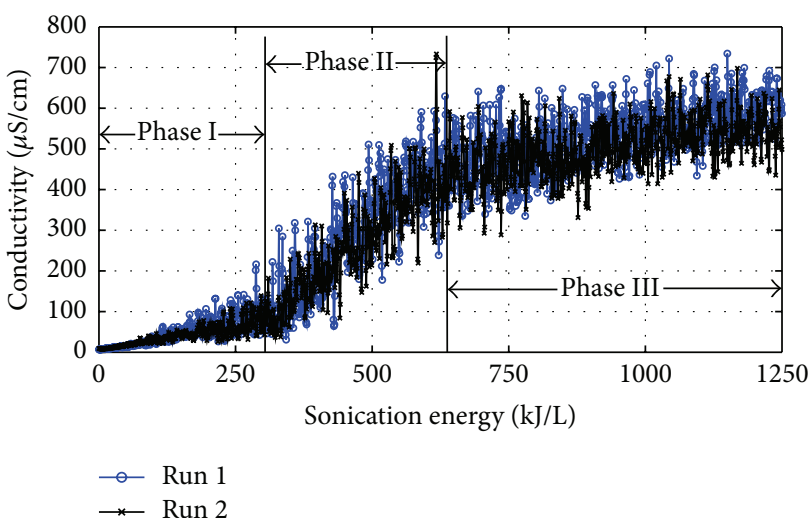

FIGURE 7: Effect of sonication energy on the electrical conductivity of $1.11 \mathrm{~g} / \mathrm{L}$ CNT solution.

energy input by the sonicator that is converted into heat can be shown to be approximately $107 \mathrm{~W}$.

Figure 7 shows the change in the electrical conductivity of the solution with the progress of the sonication process when $1.11 \mathrm{~g} / \mathrm{L} \mathrm{CNT}$ is used. It is clear from the figure that there is a steady increase in the conductivity with sonication. The abscissa here is the sonication energy, which is the output of $750 \mathrm{~W}$ sonicator employed at $100 \%$ amplitude. A close look on the figure reveals the presence of three clearly identifiable phases of mixing as demarcated in the figure. During Phase I, that is, the initial stage of mixing, conductivity slowly but steadily increases with sonication. During the second stage of sonication, identified as Phase II in the figure, there is a rather pronounced increase in the conductivity which rises severalfold during this stage of sonication. Fluctuations in the values of conductivity also show significant surge here. With further progress in sonication, the increasing trend of the conductivity tends to subside and attain a steady value.

An important feature of foregoing conductivity plots is fluctuations in the values of the electrical conductivity of the solution. Therefore, the characteristic parameter, intensity of fluctuations, is defined as

$$
\frac{\sqrt{\overline{{C^{\prime 2}}^{2}}}}{\langle\bar{C}\rangle},
$$

which is in fact normalized conductivity fluctuations. Here, $C^{\prime}$ is conductivity fluctuation and $\bar{C}$ is time-smoothed conductivity defined as

$$
\bar{C}=\frac{1}{t_{0}} \int_{t-(1 / 2) t_{0}}^{t+(1 / 2) t_{0}} C(s) d s
$$

where period $t_{0}$ is being long enough to give smooth averaged function. Note that time-smoothed conductivity fluctuation $\overline{C^{\prime}}=0$, while $\overline{C^{\prime 2}} \neq 0$. The parameter defined in (2) is analogous to commonly used intensity of turbulence in fluid mechanics literature [23].

The intensity of fluctuations plot, shown in Figure 8, is more revealing. The existence of three distinct phases 


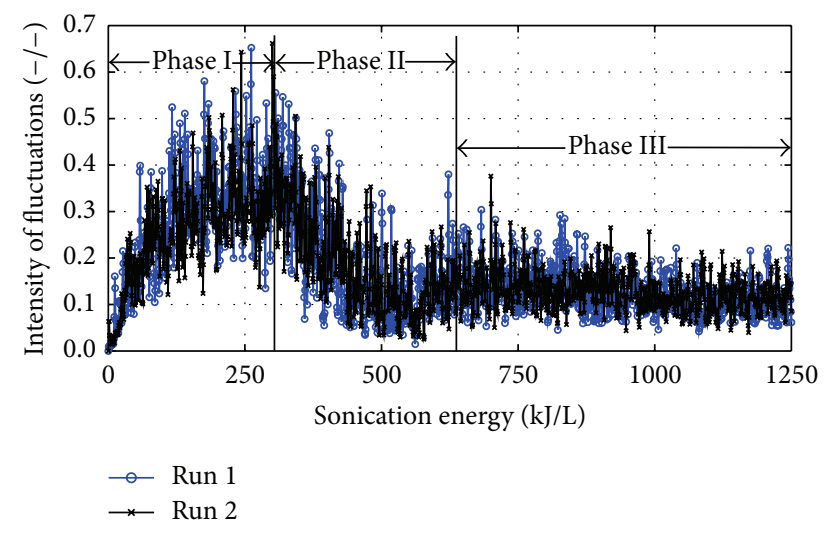

FIGURE 8: Effect of sonication on the conductivity fluctuations in $1.11 \mathrm{~g} / \mathrm{L}$ CNT solution.

corresponding to different stages of dispersion during sonication is more evident here. During Phase I, the fluctuation intensity progressively increases, ultimately attaining a maximum. During Phase II, there is a rapid decrease in the turbulent intensity. It finally attains a steady constant value of approximately 0.1 after the input of almost $650 \mathrm{~kJ} / \mathrm{L}$ of energy, which corresponds to almost 13 minutes of sonication. Further sonication hardly changes this value. The last phase of sonication where the intensity of fluctuation remains steady is identified here as the Phase III sonication.

Putting the information contained in Figures 7 and 8 together, it is clear that as sonication starts the CNTs begin to disperse in the solution so the conductivity initially shows a slow and steady increase. Since CNT agglomerates are large at this stage, their passage through the electrodes of the conductivity probe reflects in large conductivity fluctuations. The intensity of fluctuations is therefore high. During the second phase of sonication, the large CNT agglomerate starts to break down and begin dispersing in the solution. This increases the dispersion homogeneity of CNTs in the solution. As a result, the conductivity of the solution rises during the second phase of sonication, while the intensity of conductivity fluctuations shows a concomitant decrease. By the end of the second stage of the sonication, most large agglomerates have already broken down. Now the decrease in the size of agglomerates is almost negligible during the third phase of sonication. Therefore, there is a negligible increase in the conductivity. Since CNT agglomerates tend to attain a uniform size, the fluctuation intensity becomes constant during the third phase of sonication.

There were 15,000 pieces of conductivity data obtained during 25 minutes of sonication. Frequencies for all the three different phases are shown in Figure 9. The behavior of both runs is almost identical. The sharp peak for Phase III occurs at 0.1 indicating that there is narrow band of conductivity fluctuations during this phase. On the other hand, a wide distribution is seen during Phase I where conductivity fluctuations mostly range from 0.1 to 0.5 . Phase II also exhibits wide distribution, and lower magnitude fluctuations, however, dominate the behavior during this phase.
The case of $0.67 \mathrm{~g} / \mathrm{L} \mathrm{CNT} \mathrm{solution} \mathrm{is} \mathrm{shown} \mathrm{in} \mathrm{Figures}$ 10 and 11 . Here, very large fluctuations are initially observed. This can be attributed to the presence of some large flocs in the CNT sample. During the initial stages of the sonication, variation in fluctuations in different runs is expected owing to initial mixture nonhomogeneity. By the end of Phase I, corresponding to $300 \mathrm{~kJ} / \mathrm{L}$ of sonication energy, the intensity of fluctuations is almost 0.4 . During Phase II, it progressively decreases to almost 0.25 . This indicates the breakage of agglomerates, leading to greater dispersion uniformity and homogeneity of CNTs distribution in the solution. This phenomenon contributes to an almost threefold increase in the conductivity of the solution. In the third stage of sonication (Phase III), after the input of over $650 \mathrm{~kJ} / \mathrm{L}$ of sonication energy, there is negligible decrease in the intensity of fluctuations and relatively small increase in the conductivity. This means that sonication during this stage hardly contributes to the deagglomeration of CNTs.

The frequency distribution of the fluctuation intensity is shown in Figure 12. The overall trend here is the same as seen before in the previous case. A sharp and prominent peak occurs for Phase III, that is, during the later stage of sonication, while the initial phase of sonication (Phase I) shows wide distribution. During Phase II, frequency of occurrence of large fluctuations is comparable to the one of Phase I.

The case of low CNT concentration is shown in Figures 13 and 14. The trend obtained here conforms to trends obtained at higher concentrations of CNTs. During Phase I sonication, the fluctuation intensity increases attaining a maximum around 0.3. Thereafter, it shows a rather gradual decrease reaching as low as 0.1 during the later stages of Phase II sonication. The Phase III sonication does not affect the conductivity behavior of the solution. Although conductivity values in the present case are significantly lower than what was observed at higher and intermediate CNT concentrations, the fluctuation intensity nonetheless shows similar behavior with its magnitude remaining constant around 0.1 during the Phase III sonication. The frequency of occurrence of fluctuations of different intensities is shown in Figure 15. The trend is the same as seen before. Once again, the wide distribution is seen for Phase I, while Phase III as usual exhibits narrow distribution.

Once the process of sonication was over, the particle size analysis of the postprocessed CNTs sample was carried out again. Results are shown in Figure 16. The volume mean diameters are $29.89,31.91$, and $32.20 \mu \mathrm{m}$, respectively, with standard deviations being $10.69,11.94$, and $12.22 \mu \mathrm{m}$, respectively. Thus, sonication helped reduce the average agglomerate diameter by one-half. Even more significant is its effect on the size distribution as the standard deviation of the postprocessed sample is reduced by almost one-third as compared to the one of the "as-received" sample. It should however be noted here that the mutual interaction of CNTs and CNT solution outside the sonication vessel, where pressure waves are nonexistent, will be entirely different from their interaction inside the sonication vessel. Therefore, size of the postprocessed sample reported here cannot be considered a true representation of the actual CNT agglomeration behavior under sonication. 


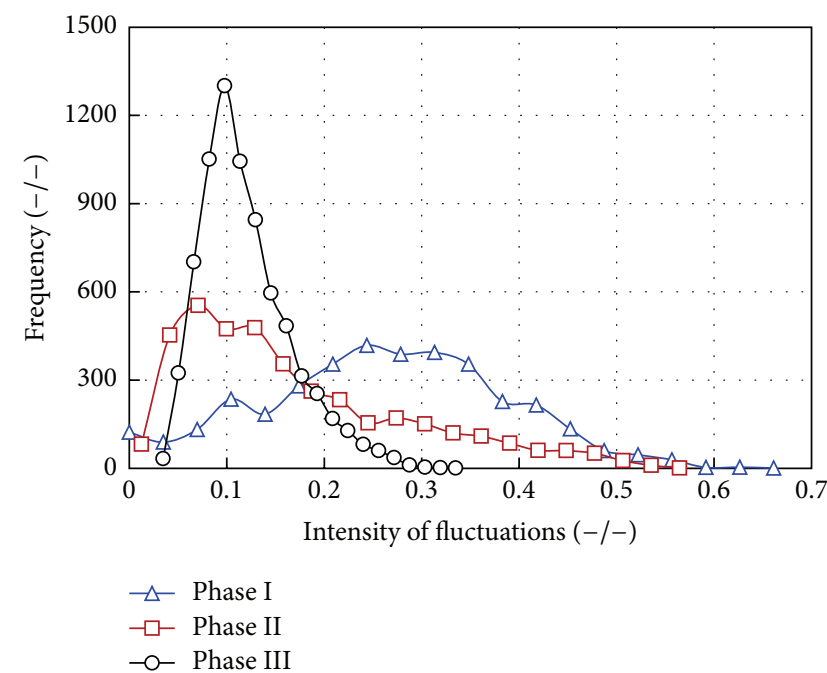

(a)

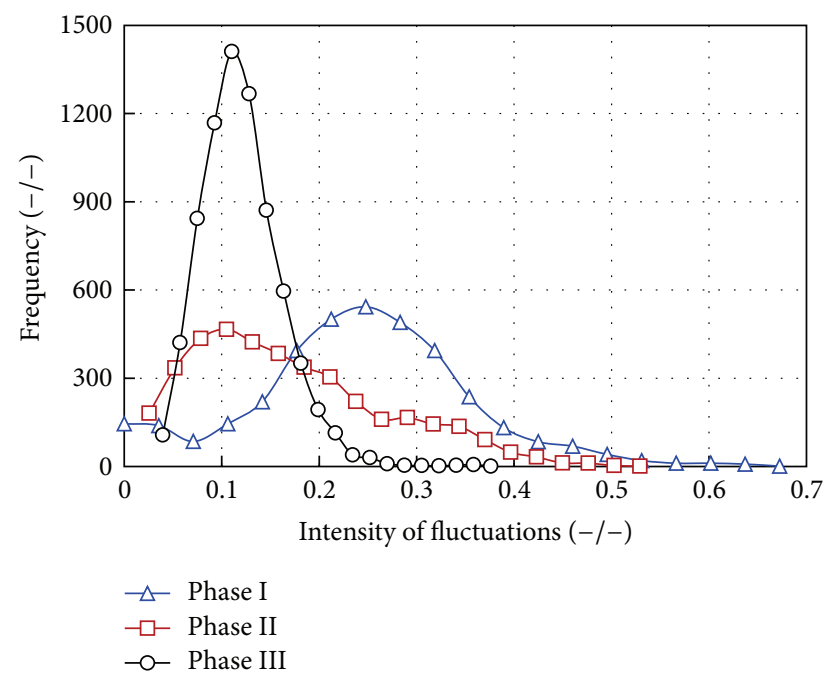

(b)

FIgURE 9: Conductivity fluctuations during different sonication phases in $1.11 \mathrm{~g} / \mathrm{L}$ CNT solution for (a) Run 1 and (b) Run 2.

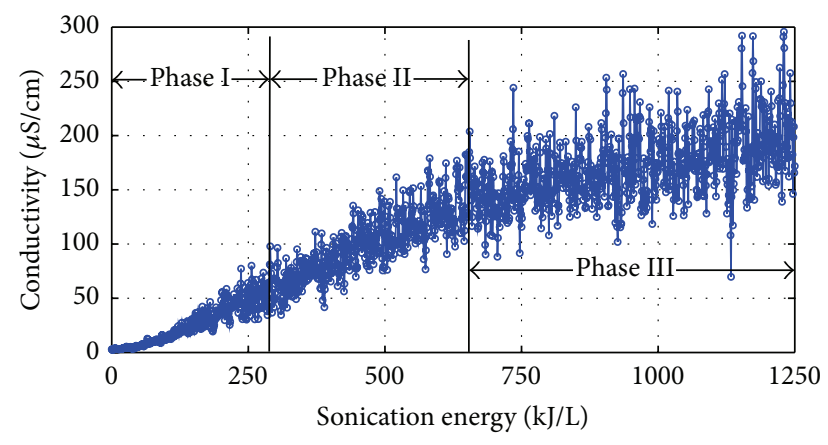

FIGURE 10: Effect of sonication energy on the electrical conductivity of $0.67 \mathrm{~g} / \mathrm{L} \mathrm{CNT}$ solution.

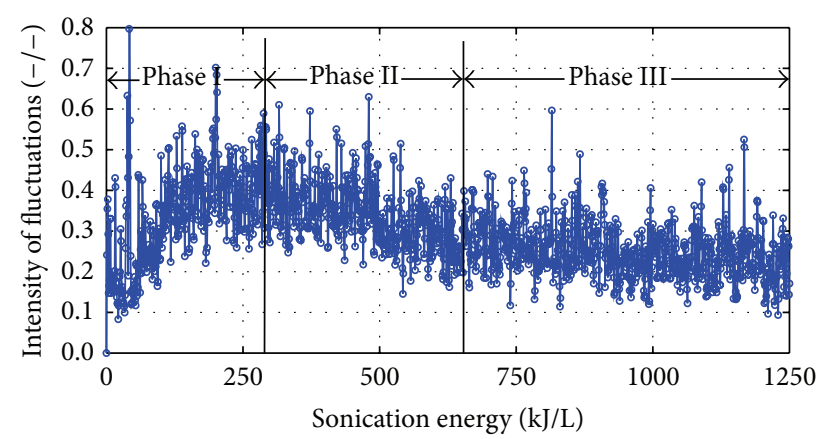

FIGURE 11: Effect of sonication on the conductivity fluctuations in $0.67 \mathrm{~g} / \mathrm{L}$ CNT solution.

\section{Conclusion}

It is clear from the results obtained here that the optimum sonication energy requirement for the CNT dispersion is approximately $650 \mathrm{~kJ} / \mathrm{L}$ of the solution irrespective of the CNT concentration. This is about 13 minutes of sonication

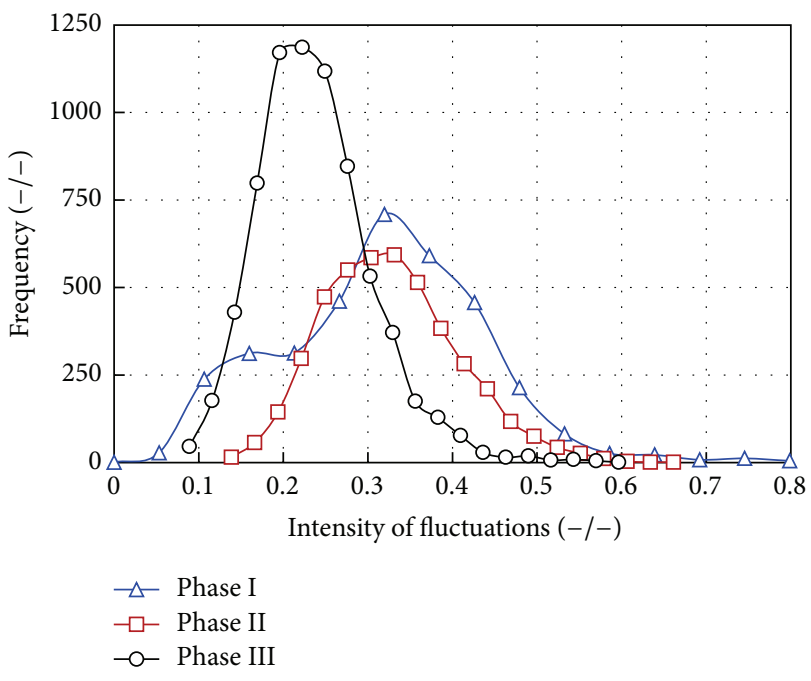

FIGURE 12: Conductivity fluctuations during different phases in $0.67 \mathrm{~g} / \mathrm{L}$ CNT solution.

using $750 \mathrm{~W}$ sonicator. It is worthwhile to note that our sonication energy requirement is close to the results obtained by Battisti et al. [19] who used sonicators of much lower power, namely, $20 \mathrm{~W}, 50 \mathrm{~W}$, and $100 \mathrm{~W}$, for much longer duration for a polymer-CNT solution of much higher viscosity.

Moreover, the transition from Phase I to Phase II occurs after $300 \mathrm{~kJ} / \mathrm{L}$ of the sonication energy. This value remains almost the same for all the three different concentrations of CNTs considered here. Although both phases exhibit wide distribution of intensity of fluctuations, yet there is a marked difference as far as dispersion dynamics in these phases are concerned. Phase I behavior arises mainly due to initial homogenization of the mixture. On the other hand, Phase II behavior reflects beginning of deagglomeration process as results of sonication. The constant value of fluctuation 


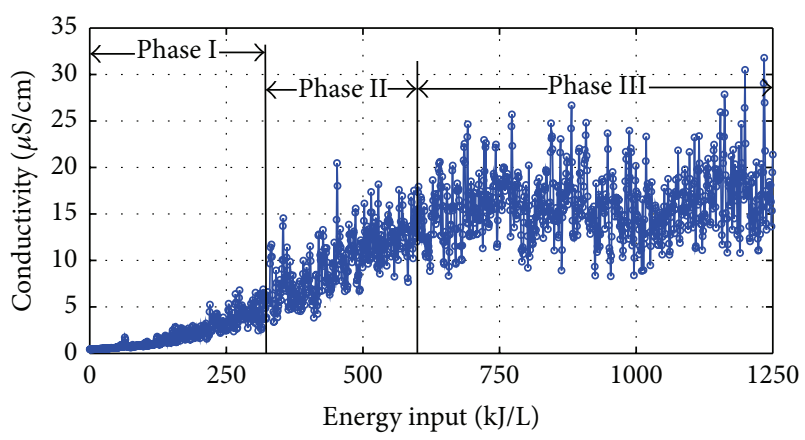

FIGURE 13: Effect of sonication energy on the electrical conductivity of $0.33 \mathrm{~g} / \mathrm{L}$ CNT solution.

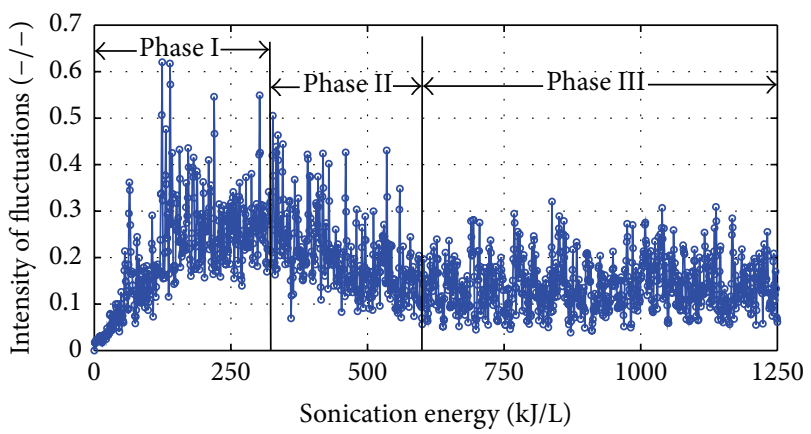

Figure 14: Effect of sonication on the conductivity fluctuations in $0.33 \mathrm{~g} / \mathrm{L}$ CNT solution.

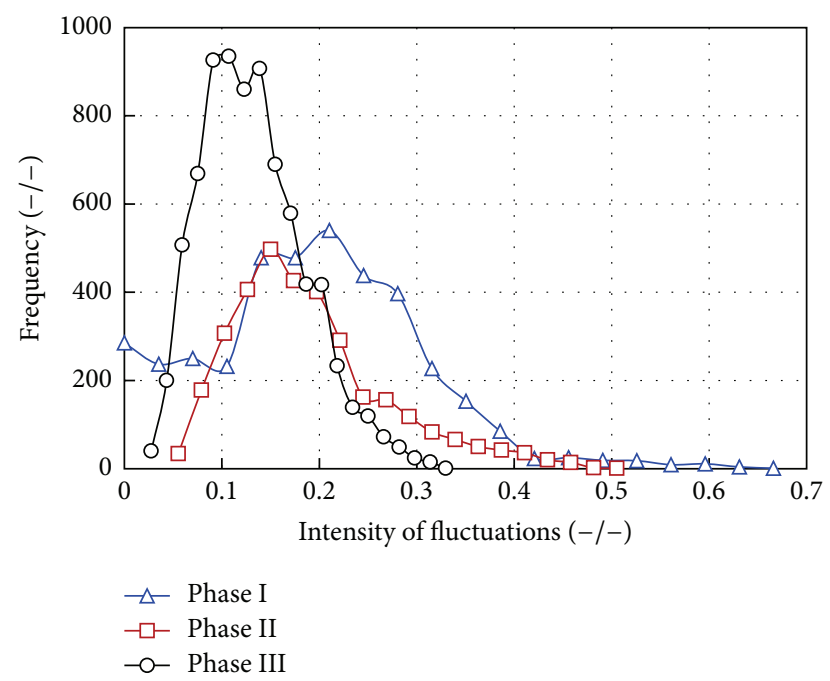

FIGURE 15: Conductivity fluctuations at each phase in $0.33 \mathrm{~g} / \mathrm{L} \mathrm{CNT}$ solution.

intensity at the end of the Phase II sonication signals the end of any further size reduction of CNTs present in the solution. However, the existence of a dynamic equilibrium deagglomeration and reaggregation process cannot be ruled out once Phase II is over.

It is also seen that the solution conductivity variation with CNT concentration is not linear. For example, although

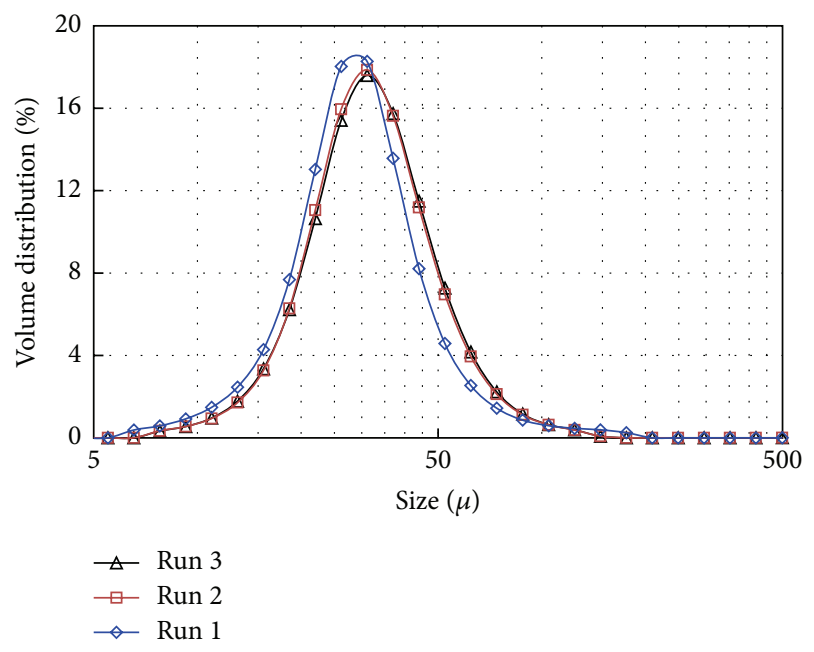

FIGURE 16: Particle size distribution of postprocessed multiwall carbon nanotube (MWCNT) sample.

the increase in CNT concentration is 3 times, there is a 30 fold conductivity increase in the solution conductivity. It is however interesting to note that the value of the intensity of conductivity fluctuations remains nearly unchanged irrespective of such a big change in solution conductivity. This indicates that as long as the temperature and the power input remain the same, the size of CNT agglomerates will also remain irrespective of CNT concentration in the solution.

\section{Conflict of Interests}

The authors declare that there is no conflict of interests regarding the publication of this paper.

\section{Acknowledgment}

The authors extend their appreciation to the Deanship of Scientific Research at King Saud University for funding this work through Research Group no. RGP-VPP-292.

\section{References}

[1] A. Montazeri and M. Chitsazzadeh, "Effect of sonication parameters on the mechanical properties of multi-walled carbon nanotube/epoxy composites," Materials \& Design, vol. 56, pp. 500-508, 2014.

[2] Y. Y. Huang and E. M. Terentjev, "Dispersion of carbon nanotubes: mixing, sonication, stabilization, and composite properties," Polymers, vol. 4, no. 1, pp. 275-295, 2012.

[3] J. Suave, L. A. F. Coelho, S. C. Amico, and S. H. Pezzin, "Effect of sonication on thermo-mechanical properties of epoxy nanocomposites with carboxylated-SWNT," Materials Science and Engineering A, vol. 509, no. 1-2, pp. 57-62, 2009.

[4] P. Pötschke, B. Krause, S. T. Buschhorn et al., "Improvement of carbon nanotube dispersion in thermoplastic composites using a three roll mill at elevated temperatures," Composites Science and Technology, vol. 74, pp. 78-84, 2013. 
[5] Y. Y. Huang, S. V. Ahir, and E. M. Terentjev, "Dispersion rheology of carbon nanotubes in a polymer matrix," Physical Review B: Condensed Matter and Materials Physics, vol. 73, no. 12, Article ID 125422, 2006.

[6] B. Munkhbayar, M. J. Nine, S. Hwang et al., "Effect of grinding speed changes on dispersibility of the treated multi-walled carbon nanotubes in aqueous solution and its thermal characteristics," Chemical Engineering and Processing: Process Intensification, vol. 61, pp. 36-41, 2012.

[7] F. Inam, A. Heaton, P. Brown, T. Peijs, and M. J. Reece, "Effects of dispersion surfactants on the properties of ceramic-carbon nanotube (CNT) nanocomposites," Ceramics International, vol. 40, no. 1, pp. 511-516, 2014.

[8] L. H. Esposito, J. A. Ramos, and G. Kortaberria, "Dispersion of carbon nanotubes in nanostructured epoxy systems for coating application," Progress in Organic Coatings, vol. 77, no. 9, pp. 1452-1458, 2014.

[9] X. Ling, Y. Wei, L. Zou, and S. Xu, "Functionalization and dispersion of multiwalled carbon nanotubes modified with poly-l-lysine," Colloids and Surfaces A: Physicochemical and Engineering Aspects, vol. 443, pp. 19-26, 2014.

[10] Q. Cheng, S. Debnath, E. Gregan, and H. J. Byrne, "UltrasoundAssisted swnts dispersion: effects of sonication parameters and solvent properties," Journal of Physical Chemistry C, vol. 114, no. 19, pp. 8821-8827, 2010.

[11] Q. Li, J. S. Church, A. Kafi, M. Naebe, and B. L. Fox, "An improved understanding of the dispersion of multi-walled carbon nanotubes in non-aqueous solvents," Journal of Nanoparticle Research, vol. 16, article 2513, 2014.

[12] T. Yamamoto, Y. Miyauchi, J. Motoyanagi et al., "Improved bath sonication method for dispersion of individual single-walled carbon nanotubes using new triphenylene-based surfactant," Japanese Journal of Applied Physics, vol. 47, no. 4, pp. 2000-2004, 2008.

[13] H. H. Le, M. N. Sriharish, S. Henning et al., "Dispersion and distribution of carbon nanotubes in ternary rubber blends," Composites Science and Technology, vol. 90, pp. 180-186, 2014.

[14] H. Yu, S. Hermann, S. E. Schulz, T. Gessner, Z. Dong, and W. J. $\mathrm{Li}$, "Optimizing sonication parameters for dispersion of singlewalled carbon nanotubes," Chemical Physics, vol. 408, pp. 11-16, 2012.

[15] P. Pötschke, A. R. Bhattacharyya, and A. Janke, "Melt mixing of polycarbonate with multiwalled carbon nanotubes: microscopic studies on the state of dispersion," European Polymer Journal, vol. 40, no. 1, pp. 137-148, 2004.

[16] Y. Y. Huang, T. P. J. Knowles, and E. M. Terentjev, "Strength of nanotubes, filaments and nanowires from sonication-induced scission," Advanced Materials, vol. 21, no. 38-39, pp. 3945-3948, 2009.

[17] U. Staudinger, B. Krause, C. Steinbach, P. Pötschke, and B. Voit, "Dispersability of multiwalled carbon nanotubes in polycarbonate-chloroform solutions," Polymer, vol. 55, no. 24, pp. 63356344, 2014.

[18] Y. Y. Huang and E. M. Terentjev, "Dispersion and rheology of carbon nanotubes in polymers," International Journal of Material Forming, vol. 1, no. 2, pp. 63-74, 2008.

[19] A. Battisti, A. A. Skordos, and I. K. Partridge, "Monitoring dispersion of carbon nanotubes in a thermosetting polyester resin," Composites Science and Technology, vol. 69, no. 10, pp. 1516-1520, 2009.
[20] A. Mohamad, "Myth about nano-fluid heat transfer enhancement," International Journal of Heat and Mass Transfer, vol. 86, pp. 397-403, 2015.

[21] http://www.cheaptubes.com/product/short-multi-walled-carbon-nanotubes-8-15nm/.

[22] J. S. Taurozzi, V. A. Hackley, and M. R. Wiesner, CEINT/NIST Protocol for Preparation of Nanoparticle Dispersions from Powdered Material Using Ultrasonic Disruption, Version 1.1, 2010.

[23] R. B. Bird, W. E. Stewart, and E. N. Lightfoot, Transport Phenomena, John Wiley \& Sons, New York, NY, USA, 2nd edition, 2007. 

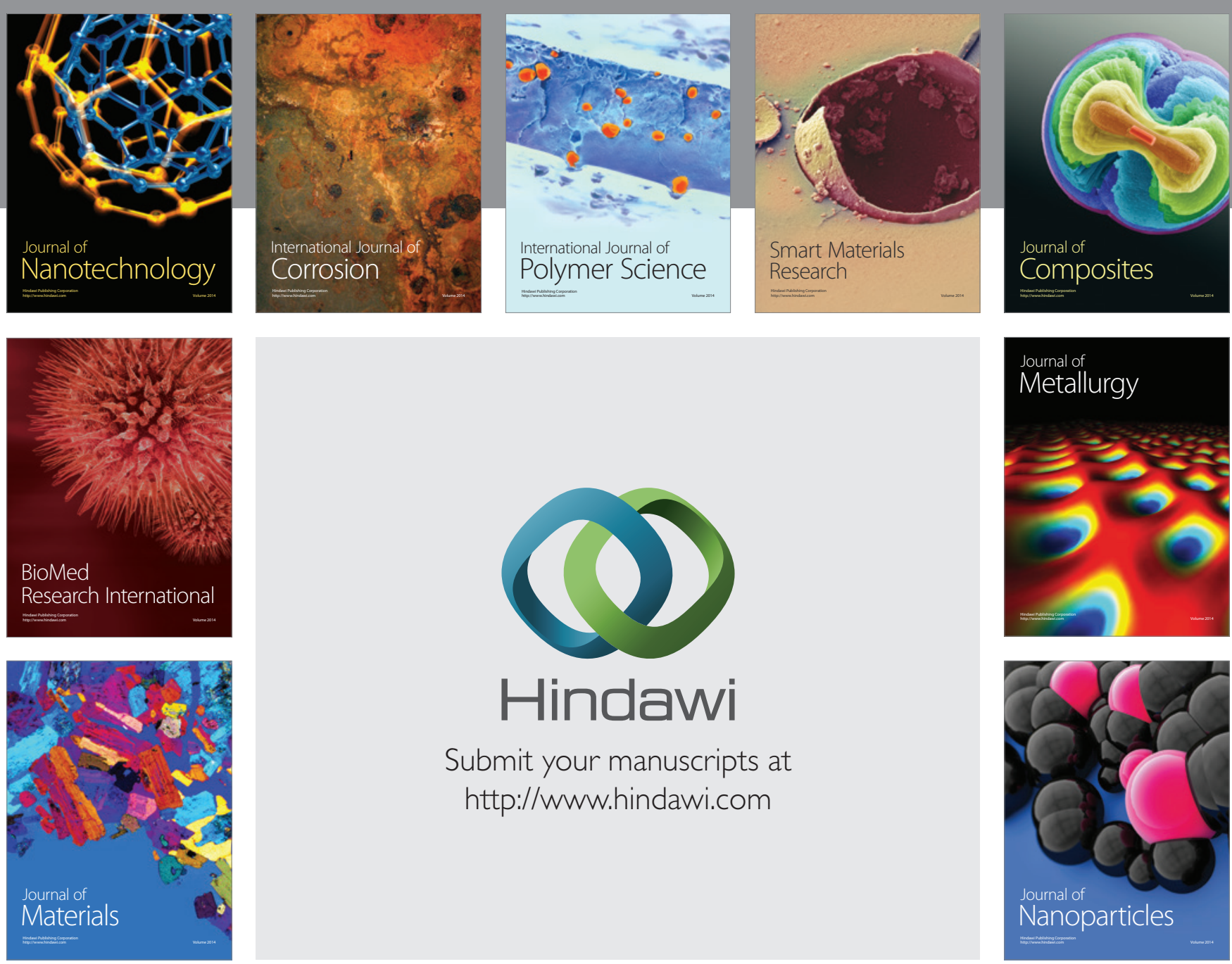

Submit your manuscripts at http://www.hindawi.com
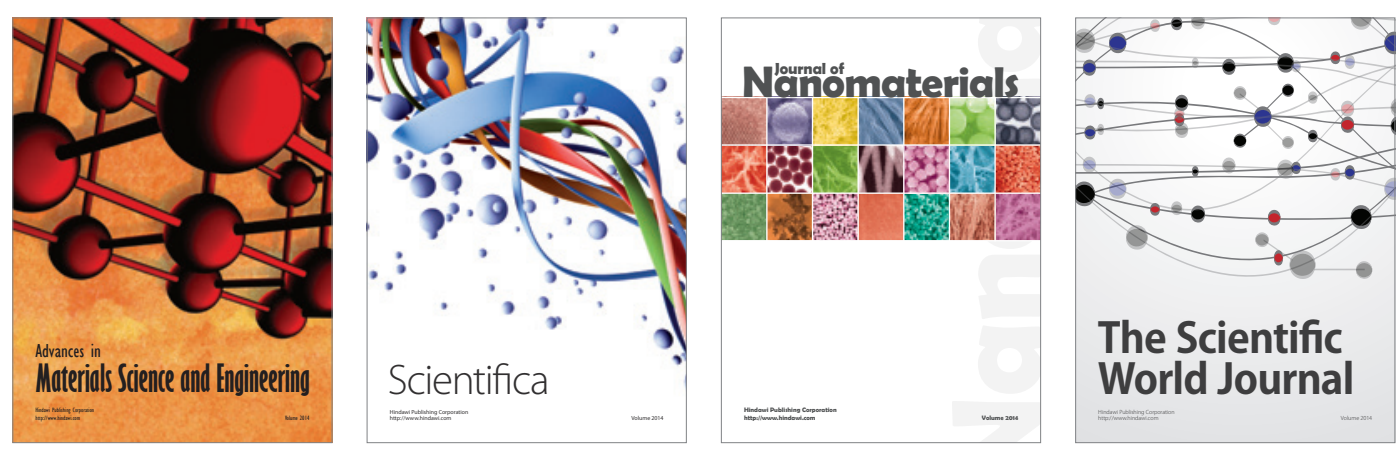

\section{The Scientific World Journal}
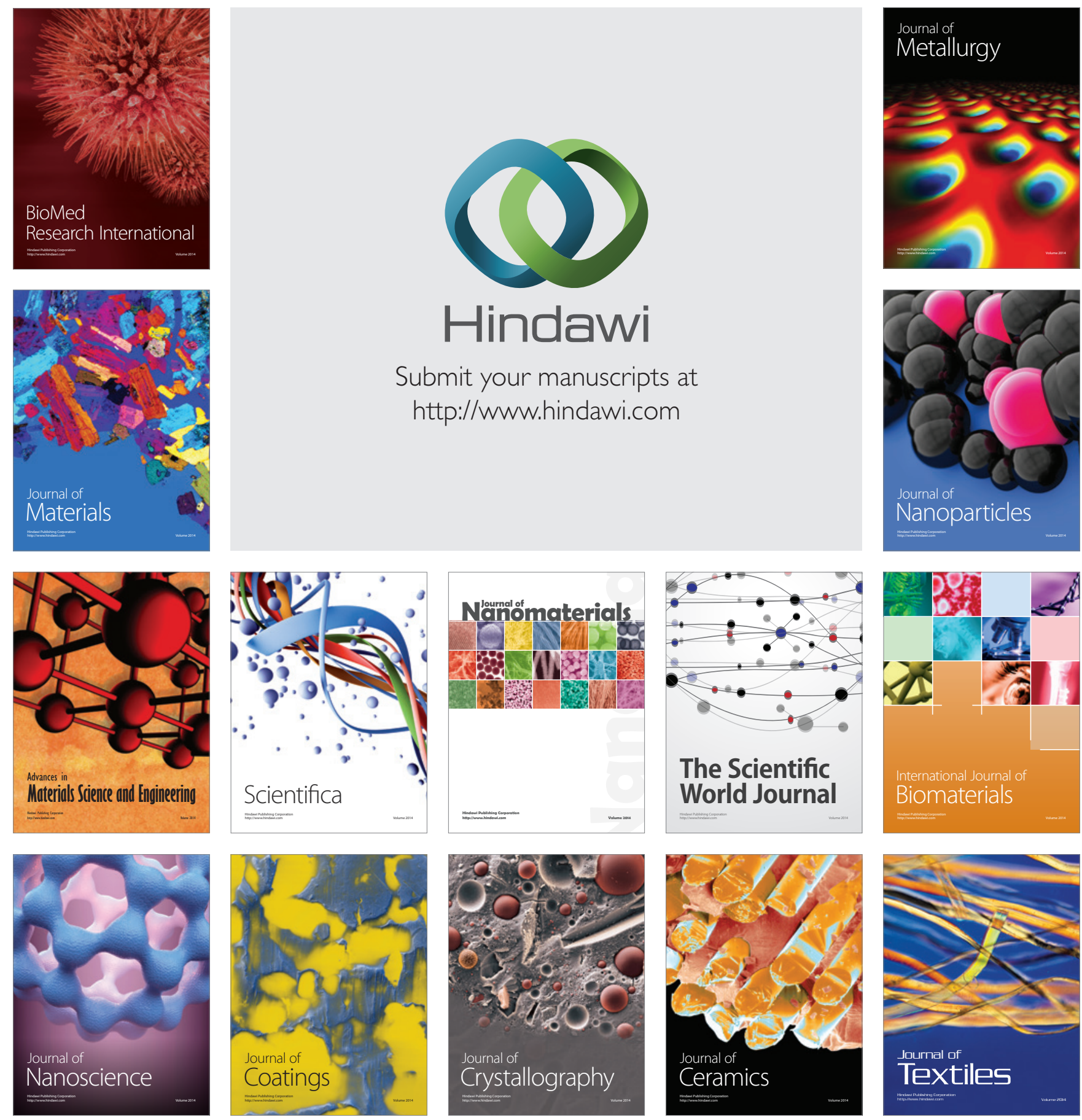